See discussions, stats, and author profiles for this publication at: https://www.researchgate.net/publication/282244106

\title{
Age-related alterations in the activation of trunk and lower limb muscles during walking
}

Article in Journal of Back and Musculoskeletal Rehabilitation · September 2015

DOI: 10.3233/BMR-150628

CITATIONS

8 authors, including:

a Nise R Marques

São Paulo State University

63 PUBLICATIONS 258 CITATIONS

SEE PROFILE

A Luciano Fernandes Crozara

2. Faculdade de Medicina de Marília

32 PUBLICATIONS 96 CITATIONS

SEE PROFILE
READS

216

Deborah Hebling

São Paulo State University

24 PUBliCATIONS 47 CITATIONS

SEE PROFILE

Mary Hellen Morcelli

33 PUBLICATIONS 139 CITATIONS

SEE PROFILE

Some of the authors of this publication are also working on these related projects:

Análise de parâmetros neuromusculares e cinemáticos dos chutes Bandal Chagui e Dolho Chagui do Taekwondo View project

Características da preensão manual de judocas de alto nível View project 


\title{
Age-related alterations in the activation of trunk and lower limb muscles during walking
}

\author{
Nise Ribeiro Marques ${ }^{\mathrm{a}, *}$, Camilla Zamfolini Hallal ${ }^{\mathrm{b}}$, Deborah Hebling Spinoso ${ }^{\mathrm{a}}$, \\ Luciano Fernandez Crozara $^{c}$, Mary Hellen Morcellic ${ }^{\mathrm{c}}$, Aline Harumi Karukad, \\ Marcelo Tavella Navega ${ }^{\mathrm{a}}$ and Mauro Gonçalves ${ }^{\mathrm{c}}$ \\ ${ }^{a}$ Department of Physical Therapy and Occupational Therapy, São Paulo State University, Marília, Brazil \\ ${ }^{\mathrm{b}}$ Department of Physical Therapy, Uberlandia Federal University, Uberlandia, Brazil \\ ${ }^{\mathrm{c}}$ Department of Physical Education, São Paulo State University, Rio Claro, Brazil \\ ${ }^{\mathrm{d}}$ Department of Physical Therapy, São Paulo State University, Presidente Prudente, Brazil
}

\begin{abstract}
.
BACKGROUND: Walking is a complex motor task that requires an integrated coordination of the trunk, lower limb, and upper limb movements. Previously, few studies have investigated the activation pattern of trunk muscles during walking. However, the mechanisms by how aging affects the recruitment of trunk muscles during walking remain unclear.

OBJECTIVE: The present study aimed to compare the activation of trunk and lower limb muscles during walking in younger and older women.

METHODS: Fifteen younger women between 18 and $30 \mathrm{yr}$ and 19 older women between 60-82 yr walked on the treadmill at a self-selected speed, while 1-min surface electromyography (EMG) signals were recorded from the multifidus, internal oblique, gluteus maximus, rectus femoris, biceps femoris, tibialis anterior, and gastrocnemius lateralis. EMG signals were processed and a linear envelope was calculated at an initial stance ( $50 \mathrm{~ms}$ after heel contact) and final stance ( $50 \mathrm{~ms}$ before toe-off).

RESULTS: Compared with younger women, older women had $52.32 \%$ lower activation of the internal oblique $(p=0.027)$ and $39.95 \%$ lower activation of the rectus femoris $(p=0.003)$ at initial stance.

CONCLUSION: Results of this study demonstrated that older women had lower activation of trunk and knee muscles during the initial stance, which may have resulted from weakness and balance impairments caused by aging.
\end{abstract}

Keywords: Aging, gait, electromyography, trunk stability

\section{Introduction}

Falls in older adults incur significant costs to the healthcare system and are responsible for approximately $50 \%$ of injuries causing death in people older than 65 years $[1,2]$. Up to $70 \%$ of falls in older adults occur during walking [3]. Thus, the differences in gait biomechanics in older patients who experienced a fall and controls have been previously investigated [4-7].

* Corresponding author: Nise Ribeiro Marques, Departamento de Fisioterapia e Terapia Ocupacional, Universidade Estadual Paulista, Avenida Hygino Muzzi Filho, 737, Mirante, CEP 13525-000, Marília, SP, Brazil. E-mail: nisermarques@yahoo.com.br.
However, a majority of these studies focused on the kinematics of the lower limbs [4-6].

Walking is a complex motor task requiring an integrated coordination of the trunk, lower limb, and upper limb movements [8]. Furthermore, the head, arms, and trunk represent more than $50 \%$ of the body mass and greatly influence the dynamic stability of the body [9]. Thus, it is suggested that the neuromuscular control of trunk motion may be prioritized over that of other inferior segments to maintain stability during movements, such as walking [9].

Aging reduces the dynamic stability across all segments, and especially in trunk motion [9]. According to Kang and Dingwell [9], the greater inertia of the trunk 
may attenuate the effect of a given perturbation on trunk motion, making feedback control less effective and reducing the ability to regain stability after a balance perturbation [9]. Further, Menz et al. [3] demonstrated that older people at the risk of falling have difficulty in controlling the rhythmic displacements of the trunk during walking. However, to our knowledge, no study has investigated the impact of aging on the recruitment of the trunk muscles [3].

Few studies have described the activation pattern of trunk and lower limb muscles during walking [10,11]. Anders et al., verified the extent of trunk muscle activation during walking at different speeds in younger healthy adults $[10,11]$. These studies showed that trunk muscles had two functions during gait: execute intervertebral and pelvic movements, required to maintain balance and reduce the metabolic cost of walking, and stabilize spinal and pelvic joints in the stance phase [10,11]. Schmitz et al. [12] investigated agerelated differences in lower limb muscle activation and demonstrated that older adults have greater coactivation of ankle muscles in mid-stance [12].

Considering that trunk and lower limb muscles may play an important role in maintaining postural control and balance, knowledge of how aging affects the recruitment pattern of these muscles could have important clinical application in preventing falls in older adults. Our study aimed to compare the activation of trunk (multifidus [MU] and internal oblique [IO]) and lower limb (gluteus maximus [GM], rectus femoris $[\mathrm{RF}]$, biceps femoris $[\mathrm{BF}]$, gastrocnemius lateralis [GL], and tibialis anterior [TA]) muscles between younger and older women in the stance phase of gait. We hypothesized that older women would have lower activation of lower limb and trunk muscles, possibly associated with age-related weakness and balance impairments.

\section{Methods}

\subsection{Participants}

Data from 34 women were considered in this prospective study (Table 1). Fifteen younger women (age, 18-30 years) were recruited from a university setting and 19 older women (60-82 years) were recruited from community-based physical activity groups. To determine the sample size, we considered the mean and standard deviation of IO activation during the stance phase obtained in the pilot study ( $n=14$, in each group; effect size $=1.3$, power $=0.95, \alpha$-error $=$ $0.05)$.

The subjects were invited to participate in the study by personal contact and provided written informed consent as approved by the Institutional Ethics Committee. People who had uncontrolled cardiovascular disease ( 2 patients with heart failure), dementia or cognitive impairment (defined as a Mini-Mental State Examination score $<20$ ), balance disturbance (defined as a BERG balance score $<36$ ), hemiparesis, pain in the lower limbs or trunk, or a progressive motor disorder were excluded.

\subsection{Procedure}

The volunteers visited the laboratory on two separate occasions within 24-72 h. On Day 1 of data collection, the preferred overground and treadmill walking speeds (PTWS) were determined. On Day 2, volunteers were familiarized with treadmill walking at the PTWS (10 min), which was followed by recording of surface electromyography (EMG) signals.

Electromyographic activity was assessed using an 8-channel, telemetered electromyogram (Noraxon ${ }^{\circledR}$, Phoenix, AR, USA), with signals recorded at a sample frequency of $2000 \mathrm{~Hz}$ using silver/silver chloride $(\mathrm{Ag} / \mathrm{AgCl})$ disc electrodes (Miotec ${ }^{\circledR}$, Porto Alegre, Brazil) with an active area of $1 \mathrm{~cm}^{2}$ and inter-electrode distance of $2 \mathrm{~cm}$ arranged in a bipolar configuration. The electrodes were positioned over muscles on the participants' right side as follows: IO, $2 \mathrm{~cm}$ medial and inferior to the anterior superior iliac spine; MU, $2 \mathrm{~cm}$ lateral to the space between the L4-L5 spinous processes; GM, midpoint of the line between the sacral vertebrae and the greater trochanter; $\mathrm{BF}$, midpoint of the line between the ischial tuberosity and the lateral epicondyle of the tibia; RF, midpoint of the line from the anterior superior iliac spine to the superior border of the patella; TA, at one third of the line between the proximal head of the fibula and the tip of the medial malleolus; and GL, at of the line between the proximal head of the fibula and the calcaneus [13,14]. A reference electrode was placed on the right medial malleolus. Before placing the electrodes, the subject's skin was shaved and cleaned with alcohol to reduce impedance [14]. Heel contact and toe-off were detected by a footswitch (Noraxon ${ }^{\circledR}$, Phoenix, USA), composed prising four sensors attached to right foot at the heel, first and fifth metatarsals, and toe, which was synchronized with the EMG system. The lower limb dominant side was assessed by the Hoffman and Payne protocol [15]. 
Table 1

Subject characteristics

\begin{tabular}{lccc}
\hline Variables & Young women group $(n=15)$ & Older women group $(n=19)$ & P-value \\
\hline Age (years) & $22.13(2.58)$ & $68.21(7.44)$ & $<0.001^{\dagger}$ \\
Mass $(\mathrm{kg})$ & $59.62(3.6)$ & $64.94(7.75)$ & $0.016^{*}$ \\
Height $(\mathrm{m})$ & $1.63(0.06)$ & $1.53(0.04)$ & $<0.001^{\dagger}$ \\
BMI $\left(\mathrm{kg} / \mathrm{m}^{2}\right)$ & $22.44(1.39)$ & $27.69(3.73)$ & $<0.001^{\dagger}$ \\
Walking speed $(\mathrm{m} / \mathrm{s})$ & $1.42(0.07)$ & $1.08(0.19)$ & $<0.001^{\dagger}$ \\
\hline
\end{tabular}

$\dagger=$ difference between groups, $P<0.01$; $^{*}=$ between-group differences, $P<0.05$; BMI $=$ body mass index.

\subsection{Gait analysis}

Preferred overground walking speed, calculated by dividing the distance walked $(10 \mathrm{~m})$ with the time to cover this distance (s), was determined on Day 1 of data collection. Volunteers were instructed to walk at their self-selected speed at a natural pace over $20 \mathrm{~m}$ and the duration for each trial was determined with 2 infrared timing gates placed at 5 and $15 \mathrm{~m}$.

The PTWS was determined by starting treadmill walking at $50 \%$ of the preferred overground speed followed by a gradual increase in speed until the subject reported that the current speed was faster than the preferred gait speed; the speed was then slowly decreased until the subject reported that the current speed was slower than the preferred gait speed. This procedure was repeated thrice, and the average of the 3 "faster" and 3 "slower" than preferred speeds was taken as the subject's PTWS. Then, the volunteers performed underwent a habituation trial involving 5 min of walking on the treadmill at the self-selected speed.

On Day 2 of data collection, volunteers were familiarized with the PTWS for $10 \mathrm{~min}$. After that, while still walking at the PTWS, 1 min of EMG data was recorded.

\subsection{Data analysis}

The EMG signal was processed according to specific routines developed in Matlab (Mathworks ${ }^{\circledR}$, Natick, USA) using a band-pass filter with a cut-off frequency of $20-500 \mathrm{~Hz}$, full-wave rectification and a low-pass, fourth-order filter with a cut-off frequency of $10 \mathrm{~Hz}$. Then, the mean of the linear envelope of the EMG signal was obtained $50 \mathrm{~ms}$ after heel contact (initial stance phase) and before toe-off (final stance phase) of in the first 10 strides. All linear envelope values were normalized to the mean activation obtained during the gait.

The PASW version 18.0 (SPSS inc.) was used for all statistical analyses. We used appropriate descriptive statistics (mean and standard deviation) to summarize participant characteristics. The Shapiro-Wilk test was

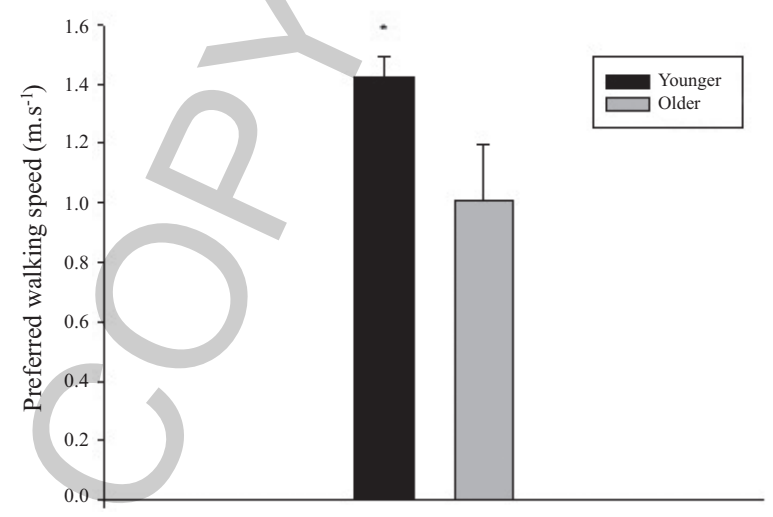

Fig. 1. Comparisons of preferred walking speed between younger and older women. ${ }^{*} P<0.05$.

used to test the normality of the data. A covariance multivariate analysis (MANCOVA) was used to compare dependent variables (muscles activation) between groups, using the gait speed as covariate. Moreover, in order to evaluate the influence of age on muscle activation, we computed the partial correlation coefficients to quantify the association between age and EMG activity, and gait velocity was factored in as a covariate. The significance level was set to $p<0.05$.

\section{Results}

Older women were heavier $(p=0.016)$, shorter $(p<0.001)$, and had a higher body mass index $(p<$ $0.001)$.

The MANCOVA analysis showed a significant main group effect $(F=2.623$ and $p=0.026)$. Younger women had $23.96 \%$ faster PTWS ( $p<0.001$; Fig. 1), $52.32 \%$ higher activation of the IO ( $p=0.001$; Fig. 2$)$, and $39.95 \%$ higher activation of the RF ( $p=0.004$; Fig. 2) at initial stance. Further, at final stance, younger women had a $57.01 \%$ higher activation of $\mathrm{BF}$ ( $p=$ 0.008 ; Fig. 2) and older women had $39.82 \%$ higher activation of TA ( $p=0.34$; Fig. 2 ). In addition, partial correlations showed that RF activation at initial stance and $\mathrm{BF}$ activation at final stance were negatively as- 

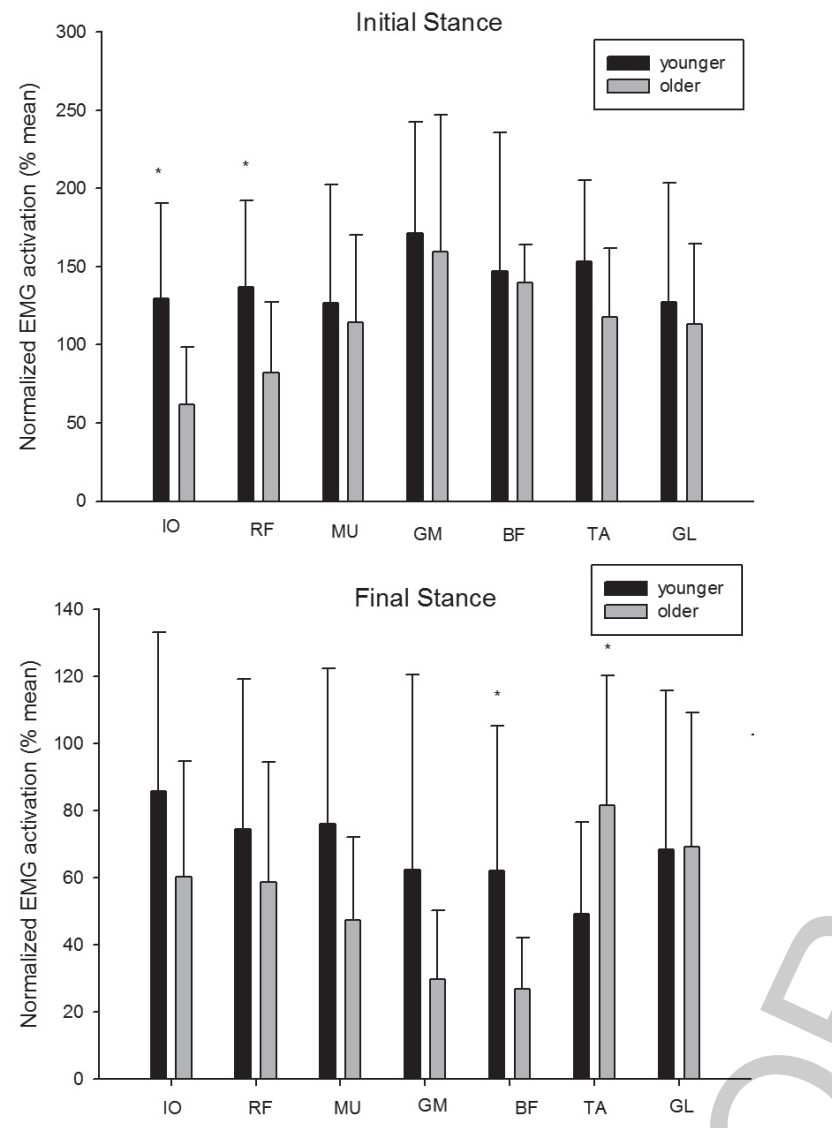

Fig. 2. Comparisons of trunk and lower limb muscle activation between younger and older women. ${ }^{*} P<0.05$.

sociated with age $(r=-0.422$ and $p=0.013$; and $r=-0.341$ and $p=0.041)$.

\section{Discussion}

The present study compared the activation of trunk (IO and MU) and lower limb (GM, RF, TA, GL, and $\mathrm{BF})$ muscles between younger and older women during the stance phase of the gait. Significant betweengroup differences were observed only during the initial stance phase for IO and RF muscles. Thus, our results partially agree with the previous hypothesis that older women would have lower trunk and lower limb muscles during the stance phase of gait.

During gait, the IO and RF have similar activation patterns, characterized by 2 peaks of activation - the first at initial stance (heel strike) and the second at final stance (push-off phase). At the initial stance, the IO is recruited to provide stability to the trunk segment, whereas the eccentric contraction of RF con- tributes to decelerate the anterior displacement of the center of mass [15]. Thus, according to our findings, older women may have impaired trunk and lower limb stability during the initial stance phase that could possibly increase the risk of falling.

In view of the fact that the proportion of active motor units is indirectly related to the force generated, we infer that older adults are more inclined to develop lower limb weakness and aging, particularly influenced by quadriceps muscles strength [16-18]. According to Ikezoe et al., the quadriceps muscles constitute the lower limb group muscle with the most lost muscle mass during aging [19]. Moreover, these authors showed that the muscle mass loss in the quadriceps has an impact on the mobility status [19].

For IO and RF muscles, the walking speed has an important contribution on the amount of EMG activation during this phase. According to Anders et al. [10], the IO activation pattern is mixed, with a continuous activation at low walking speeds $(0.55-0.83 \mathrm{~m} / \mathrm{s})$ and phasic activation at high speeds $(1.11-1.66 \mathrm{~m} / \mathrm{s})$ [10]. In addition, there is a positive association between walking speed and weight acceptance force at heel strike, which leads to an increased amount of the RF activation [20].

Our findings demonstrated that there is a negative correlation between age and walking speed, which is in concordance with the majority of previous studies [21-23]. This difference in the walking speeds between younger and older women is the main limitation of this study. Thus, to attenuate the effect of this limitation on the comparison of muscle activation between younger and older women, we considered the walking speed as covariate in the statistical analysis. In addition, from our results, we found that younger women had greater activation of BF older women had greater activation of TA at the final stance, which may contribute to the between-group difference in gait speeds.

During the final stance, the plantar flexors are highly activated to push the foot against the ground while the knee flexors and hip extensors are also activated to promote anterior acceleration of the body [15]. Thus, the higher activation of the BF in younger women may contribute to their increased walking speeds. On the other hand, the greater co-activation between the TA and GL in older women during the final stance may compromise the capacity to generate torque and accelerate the body to execute the next step [12].

Surface EMG is frequently used to describe the muscle activation pattern during human gait [10]. Recently, studies had used surface EMG to measure and describe 
trunk muscle activation during walking [10,11]. Despite the amount of fat tissues above the electrodes and the cross-talk between the muscles (IO and transversus abdominis; MU and erectors spinae), surface EMG is a noninvasive and safe method to determine spinal stability [10].

The body mass index has a strong correlation with the amount of fat tissue; therefore, in our sample, older women should have had a higher concentration of fat tissue, specifically above the areas where the electrodes were placed. Thus, the amount of EMG activation may have been reduced in this group. However, in an attempt avoid this limitation for the use of surface EMG, we amplified and normalized the signal. In addition, the history of falls in older subjects was not considered in this study; therefore, extrapolations of our results for fall prevention be made.

\section{Conclusion}

This is an initial study that investigated how age affects the EMG activation pattern of trunk and lower limb muscles during walking. The main findings of this study demonstrated that older women had lower activation of trunk and knee muscles during initial stance. Thus, according to our findings, we suggest that future studies must be conducted in order identify the effects of aging on lower limb joint and trunk stability and loading distribution during walking.

\section{Acknowledgments}

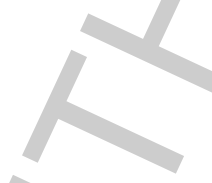

This study was funded by grants from the Fundação de Amparo à Pesquisa do Estado de São Paulo (FAPESP, 2011/11639-7), Conselho Nacional de Desenvolvimento Científico e Tecnológico (CNPq), Fundação para o Desenvolvimento da UNESP (FUNDUNESP), and Coordenação de Aperfeiçoamento de Pessoal de Nível Superior (CAPES).

\section{References}

[1] Callisaya ML, Blizard L, Schmidt MD, Martin KL, McGinley JL, Sanders LM, Srikanth VK. Gait, gait variability and the risk of multiple incident falls in older people: a populationbased. Age and Ageing 2011; 40: 481-487.

[2] Schulz BW. Healthy younger and older adults control foot placement to avoid small obstacles during gait primarily by modulating step width. J NeuroEng Rehab 2012; 9: 1-9.
[3] Menz HB, Lord RS, Fritzpatrick RC. Age-related in walking stability. Age and Ageing 2003; 32: 137-142.

[4] Gabell A, Nayak U, Wada M, Sasaki S, Kawahara H, Uchida $\mathrm{K}$. Effect of compensation procedures for velocity on repeatability and variability of gait parameters in normal subjects. Clin Rehabil 2006; 20: 239-245.

[5] Hausdorff J. Gait dynamics, fractals and falls: Finding meaning in the stride-to-stride fluctuations of human walking. Hum Mov Sci 2007; 26: 557-589.

[6] Lord S, Howe T, Greenland J, Simpson L, Rochester L (2011) Gait variability in older adults: A structured review of testing protocol and clinimetrics properties. Gait \& Posture 2011; 34 : 433-450.

[7] Marques NR, LaRoche DP, Hallal CZ, Morcelli MH, Crozara LF, Karuka AH, Navega MT, Gonçalves M. Association between energy cost of walking, muscle activation, and biomechanical parameters in older female fallers and non-fallers. Clin Biomech 2013, impress.

[8] Winter DA. Biomechanics and motor control of human movement. 3rd ed. United Kingdom: Hardcover, 2005.

[9] Kang HG, Dingwell JB. Dynamic stability of superior vs. inferior segments during walking in young and older adults. Gait Posture 2009; 30: 260-263.

[10] Anders C, Wagner H, Puta C, Grassme R, Petrovitch A, Scholle HC. Trunk muscle activation patterns during walking in different speeds. J Electromyogr Kinesiol 2007; 17: 245252.

11] Anders C, Wagner H, Puta C, Grassme R, Petrovitch A, Scholle HC. Healthy humans use sex-specific co-ordination of trunk muscles during gait. Eur J App Physiol 2009; 105: 585-594.

[12] Schmitz A, Silder A, Heiderscheit B, Mahoney J, Thelen DG. Differences in lower-extermity muscular activation during walking between healthy older and young adults. J Electromyogr Kinesiol 2008; 19: 1085-1091.

[13] Marshall P, Murphy B. The validity and reliability of surface EMG to assess the neuromuscular response of the abdominal muscles to rapid limb movement. J Electromyogr Kinesiol 2003; 13: 477-489.

[14] Hermens JH, Freriks B, Disselhorst-Klug C, Rau G. Development of recommendations for SEMG sensors and sensor placement procedures. J Electromyogr Kinesiol 2000; 10: 361-374.

[15] Vaughan CL, Davis BL, O'Connor JC. Dynamics of human gait. 2nd ed. Cape Town: Kiboho Publishers; 1998.

[16] De Luca CJ. The use of surface electromyography in biomechanics. J App Biomech 1997;13: 135-163.

[17] LaRoche DP, Cremin KA, Greenleaf B, Croce RV. Rapid torque development in older female fallers and non-fallers: A comparison across lower-extremity muscles. J Electromyogr Kinesiol 2010; 20(3): 482-8.

[18] Crozara LF, Morcelli MH, Marques NR, Hallal CZ, Spinoso DH, Almeida Neto AF, Cardozo AC, Gonçalves M. Motor readiness and joint torque production in lower limbs of older women fallers and non-fallers. J Electromyogr Kinesiol 2013, impress.

[19] Ikezoe T, Mori N, Nakamura M, Ichihashi N. Atrophy of the lower limb in elderly women: is it related to walking ability? Eur J Appl 2011;111: 989-995.

[20] LaRoche DL, Millet ED, Kralian RJ. Low strength is related with diminished ground reaction forces and walking performance in older women. Gait Post 2011; 33: 668-672.

[21] Novaes RD, Miranda AS, Dourado VZ. Usual gait speed in 
middle-aged and elderly Brazilian subjects. Br J Phys Ther 2011; 15(2): 117-122.

[22] Bohannon RW, Andrews A W, Thomas MW. Walking speed: reference values and correlates for older adults. J Orthop and
Sports Phys Ther 1996; 24(2): 86-90.

[23] Bohannon RW. Comfortable and maximum walking speed of adults aged 20-79 years: reference values and determinants. Age Aging 1997; 26(1): 15-19. 